\title{
Stéréotypage discursif d'une image de presse
}

J'aborderai la notion de stéréotypie, dans une acception commune, comme étant de l'ordre de l'idéologie (idées reçues, prêt-à-penser, doxa), de la phraséologie, qui est l'une des formes linguistiques des discours institués, et de l'iconologie, quand il s'agit de signes visuels. Quant au nom d'action "stéréotypage ", attesté pour désigner l'action de stéréotyper ${ }^{1}$ ou son résultat, et avancé par Ruth Amossy dans son étude sur la sémiologie du stéréotype ${ }^{2}$, il permet de mettre en avant un processus de stabilisation sémiotique qui tend, par la

1. Voici l'article du Trésor de la Langue Française informatisé : «STÉRÉOTYPER, verbe trans. A. - IMPR., Clicher une composition en coulant sur le flan ou empreinte de cette composition, de la matière en fusion, pour en former une planche solide (Des.-Muller Impr. 1912). B. - Au fig. 1. Rendre invariable et répéter de façon quasi-automatique. Stéréotyper des expressions. Dans leurs jeux, l'instabilité (...) est quelque peu compensée par la tendance à stéréotyper les gestes, à répéter les mêmes séquences sans se lasser (Jeux et sports, 1967, p. 79). 2. Fixer de façon immuable. L'auberge (...) avait pour maître un de ces gras et gros hommes (...) qui sont stéréotypés chez tous les romanciers (Balzac, Splend. et mis., 1844, p. 310). - Empl. pronom. réfl. Se figer. Désirs qui se sont stéréotypés. Ayant fait son ouvre, une noblesse nouvelle et une royauté nouvelle, elle [la bourgeoisie] perdit sa mobilité, se stéréotypa, et resta une classe, trop souvent ridicule (Michelet, Peuple, 1846, p. 147). Toute pensée aspire à se stéréotyper et à se poser comme éternelle (Renan, Avenir sc., 1890, p. 383). » (http://www.cnrtl.fr/definition/stéréotypage)

2. "Plutôt que de stéréotype il faudrait parler de stéréotypage. C’està-dire de l'activité qui découpe ou repère, dans le fonctionnement du réel ou du texte, un modèle collectif figé. (...) Le stéréotypage consiste en une lecture programmée du réel ou du texte. ", Amossy, Ruth, 1991, Les idées reçues. Sémiologie du stéréotype, Nathan, Paris, pp. 21-22. Voir aussi Amossy, Ruth, Herschberg, Pierrot, Anne, 2007, Stéréotypes et clichés : langue, discours, société, Armand Colin, Paris.

\section{DOMINIQUE DUCARD}

En retraçant le destin médiatique d'une photographie de presse, depuis sa fonction d'illustration d'un événement tragique de la " décennie noire du terrorisme " en Algérie jusqu'à sa transposition formelle en cuvre d'art, nous esquissons le processus sémiotique de "stéréotypage ", à la fois discursif et iconique, d'une image. Les interprétations auxquelles elle est soumise, et dont sont étudiés les formes et les mécanismes, dès les premières légendes et avec les gloses qui vont suivre, opèrent une brusque conversion du regard par subordination du sens au modèle iconographique de la tradition religieuse occidentale. À travers ce qui est figuré et ce qui en est dit, la question se pose du poids des mots sur la force des images et de l'écran culturel de la mémoire visuelle sur notre perception du monde.

Mots clés : " stéréotypage ", photographie et discours de presse, semiosis, imaginaire culturel, iconographie, transposition artistique, conversion du regard 
reprise et la réplication, au schématisme et au figement. Pour compléter cette entrée en matière sur ce qui est l'objet de cet article, à travers une étude de cas, je convoquerai des commentaires de photoreporters en Afghanistan, en 2001, qui déclaraient être réduits, en raison de la restriction imposée au droit de l'information, à photographier des «stéréotypes » de la guerre et de la souffrance. « À savoir, nous dit le journaliste qui rapporte ces propos, des photos primaires qui se répètent, par leur motif et par leurs formes, d'un conflit à l'autre; qui, sans mention de la date et du lieu, seraient interchangeables; des images qui, plutôt que d'informer ou de susciter la réflexion, renvoient le spectateur à des codes visuels qui renforcent ses convictions. $»^{3}$

Le cas que je vais présenter, en faisant porter l'accent sur le stéréotypage comme processus de construction du sens subordonné aux formes d'une mémoire culturelle, relève plutôt de ce que Michel Convert, artiste plasticien, appelle un stéréotype fort, j’y reviendrai à la fin.

\section{ILLUSTRATION}

Rappel historique : en décembre 1991, le Front Islamique du Salut arrive en tête du premier tour des élections législatives en Algérie, mais le pouvoir, anticipant une victoire possible de celui-ci, annule le scrutin. Débute alors, après l'interdiction $\mathrm{du}$ FIS et les premières arrestations, la décennie noire du terrorisme avec le conflit armé entre le gouvernement algérien et des groupes islamistes, notamment le Mouvement Islamique Armé (MIA) et le Groupe Islamique Armé (GIA). Le premier formera, en 1994, l'Armée Islamique du Salut, fidèle au FIS, tandis que le second s'opposera à celui-ci. Le conflit entre les deux groupes s'intensifie après l'élection du général Liamine Zéroual, en 1995. S'ensuit une série d'actions criminelles et de massacres dans des villages.

Dans la nuit du 22 au 23 septembre 1997, une tuerie est commise dans le village de Bentalha, à une dizaine de kilomètres au sud d'Alger. L'événement sanglant fait la « Une » de nombreux quotidiens nationaux et internationaux, le 24 septembre, avec la même photographie en illustration, en noir et blanc ou en couleurs. Le Monde fait exception, dans son édition du 25 septembre, et titre, dans la soustribune gauche, sur l'annonce par le FIS d'un appel à l'arrêt des combats, dans un communiqué de presse daté du 21 mais publié dans la presse algérienne après le massacre : "Algérie : l'aile militaire du FIS appelle au cessez-le-feu ». Le fait est développé en page deux dans la rubrique «International » : «Le bras armé du FIS ordonne un arrêt des combats. » Un article de Jean-Pierre Turquoi fait alterner récit d'une nuit d'horreur et analyse des forces et des stratégies qui s'opposent, illustré par un dessin de Sergueï.

Si l'on décrit le processus de signification comme une relation dynamique entre les éléments qui sont mis en présence, dans le contexte le plus immédiat, on peut comprendre la fonction d'illustration de la photographie, et son action sur le lecteur-interprète, en disant, si l'on se réfère à la triade de Peirce, qu'un

3. Guerrin, Michel, 2001, «Les stéréotypes visuels de la guerre en Afghanistan », Le Monde, vendredi 16 novembre. 
représentant ou representamen (photographie de presse) détermine un interprétant composite (légende, titraille, chapeau, article) à représenter l'objet que lui-même représente : douleur d'une mère algérienne qui a perdu ses enfants dans un massacre à Bentalha, selon les premières informations données ${ }^{4}$. Mais l'on peut aussi dire, en inversant l'ordre de la représentation, que le texte, en tant que représentant, détermine l'image, son interprétant, à représenter ce à quoi il renvoie, son objet. La fonction d'illustration de la photographie de presse est de donner à voir une image partielle, par sélection d'une scène, de l'événement rapporté et commenté par le texte. Le signe ainsi constitué dans cet aller-retour produit un effet de signification (connaissance, affect) dans l'esprit de l'interprète.

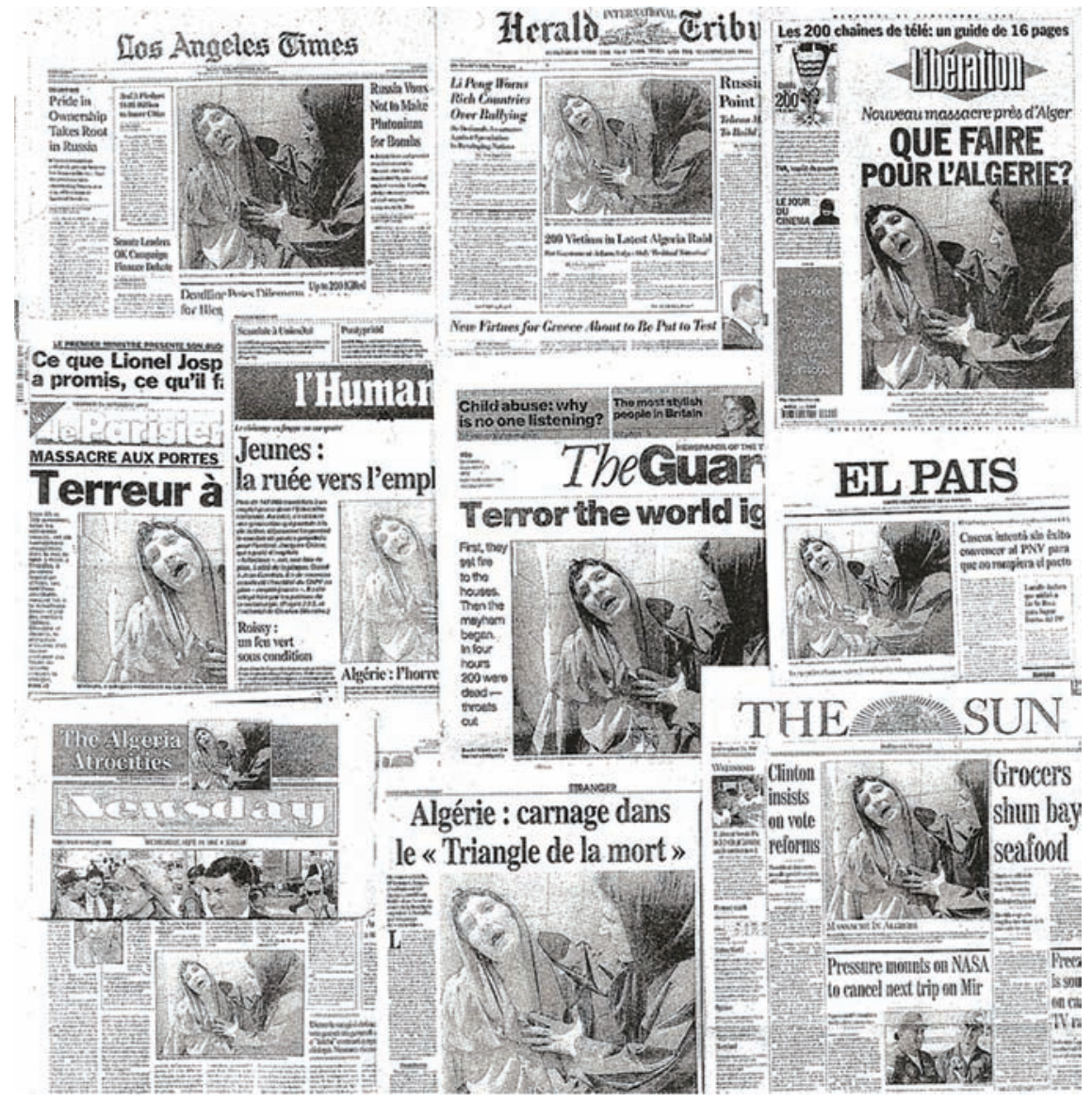

Figure 1 : la tuerie de Bentalha dans les « Unes » des 22 au 23 septembre 1997

4. Voici, par exemple, la légende du Parisien : «Bentalha. À quelques kilomètres au sud d'Alger. Hier matin ", celle de Libération: "Dans la nuit de lundi à mardi, près d'Alger, au moins deux cents civils ont été massacrés à la hache ou au couteau (photo : hier, la douleur des proches) » et celle du Figaro: « Une femme algérienne crie sa douleur après un nouveau drame ». 


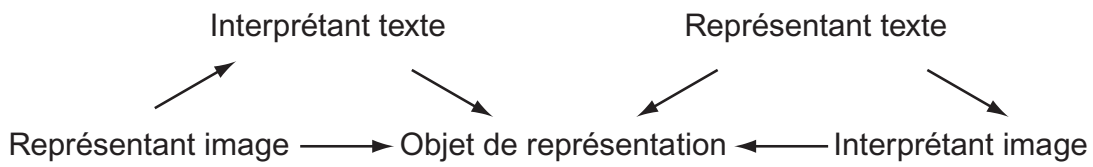

Figure 2

L'image en elle-même, sur laquelle se focalise le regard, peut susciter d'autres interprétants. C'est ainsi qu'elle fait l'objet d'un commentaire de Pierre Georges, à la fin de sa chronique, à la page 26 du même numéro du Monde (daté du 25 septembre), dans un encadré titré " Mater dolorosa ". L'illustration y est convertie en image de méditation et de contemplation, exemplaire, répondant au mutisme provoqué par l'effroi :

Il y a des jours où l'on peut regretter que Le Monde ne publie pas de photos en « une ». Toute la presse, nationale et internationale, s'est retrouvée pour illustrer ce drame permanent d'un document extraordinaire. Une simple photographie due à un reporter de l'Agence France-Presse. Mais, cette fois-ci, sans risque d'être contredit, il s'agit de LA photographie.

On y voit deux femmes algériennes, simplement, l'une soutenant l'autre, et pourtant le document touche au chef-d'œuvre. On l'a regardée encore et encore, fasciné. Qui dira mieux et plus que ce visage de mater dolorosa la cruauté de la tragédie algérienne.

\section{Figuration}

On retrouve la photographie à la « Une » du Monde du 26 septembre 1997, dans la sous-tribune gauche. Le texte, sous l'image, porte un titre qui sert aussi de légende : "Une madone en enfer». Les pages 12 et 13 sont consacrées au sujet, avec la reprise de l'image dans un format plus grand et une analyse de Michel Guerrin, qui est une enquête sur le photoreportage en temps de guerre, en référence au conflit qui ensanglante l'Algérie et à partir de l'événement d'actualité. On y retrouve les " lieux communs » d'un débat récurrent dans l'histoire du photojournalisme : la tension entre information et émotion : dénommer et décrire l'événement ou figurer et produire de l'affect; le visible et le montrable : questions de l'accès aux lieux et de l'autorisation accordée ou non aux journalistes, du contrôle et de la censure des images, pour le "visible ", question du degré d'indexicalité de l'image, de connexion avec le réel, l'impossible (Lacan), pour le "montrable ", c'est-à-dire trace directe de l'acte effectué sur les victimes ou trace indirecte de la douleur sur les proches ${ }^{5}$; le thème de l'indicible et de la faillite des mots pour dire $l^{\prime}$ horreur'.

5. "L'image est intemporelle, peu informative, mais que signifie une information dans l'Algérie d'aujourd'hui ? Quand les repères s'évanouissent, il ne reste que la douleur du témoignage, si intense dans ce cliché qui émeut davantage qu'une série de cadavres alignés, au petit matin. » (article cité)

6. «Quand l'horreur est sans limite et qu'on a épuisé les superlatifs, la photographie prend le relais et "bouffe" les premières pages. Pour éviter également la banalisation de l'ignoble.» (Ibid. propos rapporté d'un journaliste d'El Watan) 
La rhétorique discursive procède, à différents niveaux, à la fois à une universalisation et à une appropriation culturelle de l'image, selon plusieurs procédés : par une réduction métonymique à l'expression naturelle des émotions : c'est un visage, un cri, un regard, et par typification et sériation : cette mère - une mère - la Mère.

LE VISAGE de cette mère qui a perdu ses huit enfants dans le massacre, lundi 22 septembre, de Bentalha a fait la « une » dans le monde entier. Hocine, l'auteur de ce document, raconte la vie de photographe en Algérie. (en page une)

"C’EST un cri de désespoir qui a éclaboussé la « une » des journaux, mercredi 24 septembre. C'est un regard, celui d'une femme comme déjà dans l'au-delà. C'est le portrait d'une mère à genoux, qui a perdu ses enfants, soutenue par une autre mère. C'est enfin une Pietà que l'on peut rapprocher d'une riche iconographie picturale, une madone en enfer. »

À accrocher, à côté d'autres Pietà, comme cette Japonaise baignant son enfant déformé par une pollution au mercure, ou ces huit femmes au Kosovo pleurant un être cher tué par la police serbe. Il y a l'émotion et il y a les faits, brutaux, qui donnent tout leur sens à l'image algérienne. "Cette femme qui hurle vient de perdre ses huit enfants ; celle qui la soutient, ses parents. » [...] L'histoire du photojournalisme est truffée de Pietà qui pleurent leurs enfants sur les champs et ruines de la guerre.

Et l'image est élevée au rang d'un « un symbole qui appelle la compassion », selon le patron de l'agence Sipa.

\section{Symbolisation}

La dénomination, par le titre, le nom et le surnom, participe activement à cette acculturation. Le titre de l'article publié dans Le Monde et qui sert de légende à la photographie reproduite à la "Une » est : "Une madone en enfer ». Le mot " madone ", comme chacun le sait, est un emprunt à l'italien Madonna (m(i)a donna), appellatif de respect donné aux femmes, attesté depuis le $\mathrm{XI}^{\mathrm{e}}$ siècle comme désignation de la Vierge Marie, et employé dans les beaux-arts depuis le XVI ${ }^{\mathrm{e}}$ siècle. Il s'emploie généralement avec une majuscule, pour désigner une représentation de la Vierge, surtout les peintures ou sculptures italiennes, plus particulièrement les vierges à l'enfant. En référence à l'Italie ou à la Corse, La Madone - ou Madone, dans les prières et les invocations - est le nom de la Vierge Marie, et fonctionne comme un nom propre. Le journaliste du Monde l'utilise comme un nom commun, dans une alliance d'idées, presque un jeu de mots antithétiques, qui pourrait avoir, hors contexte, une connotation blasphématoire. La composition du titre ne donnera pas lieu à commentaire mais va générer, dans les multiples gloses qui vont s'ensuivre et ce jusqu'à aujourd'hui, une série de dénominations propres du sujet ou de l'image elle-même, sous des formes diverses, en référence au sujet représenté : la "madone » algérienne (Le Monde, 28 octobre 1997), la « madone de Benthala », la "pietà algérienne » (Le Monde, 18 juillet 1998), la " madone » (Le Monde, 7 octobre 2005), la « Madone » algérienne (Le Monde, 29 mai 2006) ; en référence à l'image elle-même : la «madone algérienne » (entretien avec Benjamin Stora, Le Monde, 24 avril 2001 ; entretien avec Jean Baudrillard, Le Monde, 30 août 2003), la "madone de Benthala » (Le Monde, 7 octobre 2005), la « madone » de Benthala (Le Monde, 13 octobre 2005), la « madone » d'Hocine Zaourar (Le Monde, 
7 octobre 2005), la « madone» (Ibid.), La Madone d'Hocine Zaourar (entretien avec Christian Caujolle, directeur de l'agence VU, Le Monde, 7 octobre 2005). On trouve aussi des emplois en mention qui, à la manière des guillemets, détache le nom comme un surnom : " celle que l'on surnomme la "madone de Benthala" ", " la photographie dite "madone de Benthala" ". De la nomination lors de la première apparition : "Une femme algérienne crie sa douleur après un nouveau drame » dans Le Figaro, par exemple, nous sommes passés à la qualification, à connotation religieuse : " une madone », puis à une dénomination propre : « la madone algérienne » ou « de Bentalha », requalifiée par localisation, qui vaut autant pour le sujet que pour la représentation, analogue en cela aux emplois usuels du nom « madone ». La nouvelle madone trouve alors sa place en tant qu'occurrence spécifique d'un type commun : la Mater dolorosa, dont elle est un exemplaire unique et dont elle porte le nom propre générique.

\section{FALSIFICATION}

S'est ainsi engagée une opération de déréalisation de la photographie en tant qu'illustration d'un événement historique, dans son actualité, par un dépassement de la valeur référentielle, indexée par la légende et le texte informatif, que vient doubler une valeur représentationnelle par analogie de l'image avec un type de figuration conventionnelle. La photographie de presse se trouve alors annexée à un modèle culturel appartenant à l'histoire des arts visuels, plus précisément à l'iconographie chrétienne ${ }^{7}$, relayée par l'histoire du photojournalisme. Le mouvement, apparemment paradoxal, d'universalisation et d'appropriation trouve ainsi son moyen terme et sa résolution dans une figure stéréotypée - forme figurale et figurative - qui d'une part constitue une empreinte visuelle et d'autre part assure une fonction d'interprétant final ou logique, au sens de Peirce, c'est-à-dire d'un signe dont l'interprétation est régulée par des normes collectives, acquis par habitude, et qui vaut comme symbole, signe légalisé.

Cette déréalisation de l'image photographique, associée à la surimposition d'une vision et d'un sens religieux chrétiens et à la réaction du pouvoir algérien, a conduit à l'accusation, notamment par le quotidien pro-gouvernemental Horizons, de falsification et de mise en scène. Il a fallu une autre photo, prise sous un autre angle par un journaliste d'El Watan, pour mettre fin à toute controverse, controverse habituelle en la circonstance, là où la vérité esthétique de l'image est confrontée au vraisemblable de la figuration : le trop beau pour être vrai. À cette accusation s'ajoutent les critiques, suite au démenti de la première légende, erronée : la femme n'a pas perdu ses enfants mais son frère, sa belle-sœur et son neveu. Puis ce sera la plainte, en juillet 1998, de la femme photographiée, madame Oum Saad, contre l'AFP et contre Hocine Zaourar - dont le nom de famille n'est

7. Voir Pacaut, Marcel, 1962, L’iconographie chrétienne, PUF, coll. «Que sais-je ? ». Rappelons la définition qui est donnée de l'iconographie: "'L’iconographie est l'étude des représentations figurées, soit d'un individu, soit d'une époque, soit des symboles et des dogmes d'une doctrine ou d'une religion". Alors que l'histoire de l'art s'attache aux styles, elle s'intéresse aux types, à la matière même des œuvres d'art. Ainsi, l'iconographie chrétienne est l'étude de la représentation, dans la production artistique, des éléments divers dogmatiques, liturgiques, historiques - du christianisme. » (Ibid., p. 6) 
pas encore cité par la presse alors que la nomination de la femme lui assigne une identité personnelle - pour atteinte à l'image et pour diffamation (affaire conclue par un non-lieu après cinq années de procédure). Elle avait entre-temps fait une déclaration à la presse, demandant que l'on cesse d'utiliser son image, en récusant sa désignation par le terme de "madone».

Nous avons les éléments déterminants pour le stéréotypage de la photographie, qui est détachée de la situation et de son sens référentiel (le «ça a été " de Barthes) : anonymat initial du sujet et du photographe, photogénie ${ }^{8}$ de la prise de vue - l'illustration devient une belle photographie -, application d'un modèle et d'une tradition iconographiques à la figuration, imposition d'un nom générique codifié par l'histoire biblique - le nom est quasiment un nom de code -, erreur de légende, procès en falsification et diffamation. Tous ces éléments signifiants portent à l'artifice et à la perte d'identité singulière.

\section{ESTHÉTISATION}

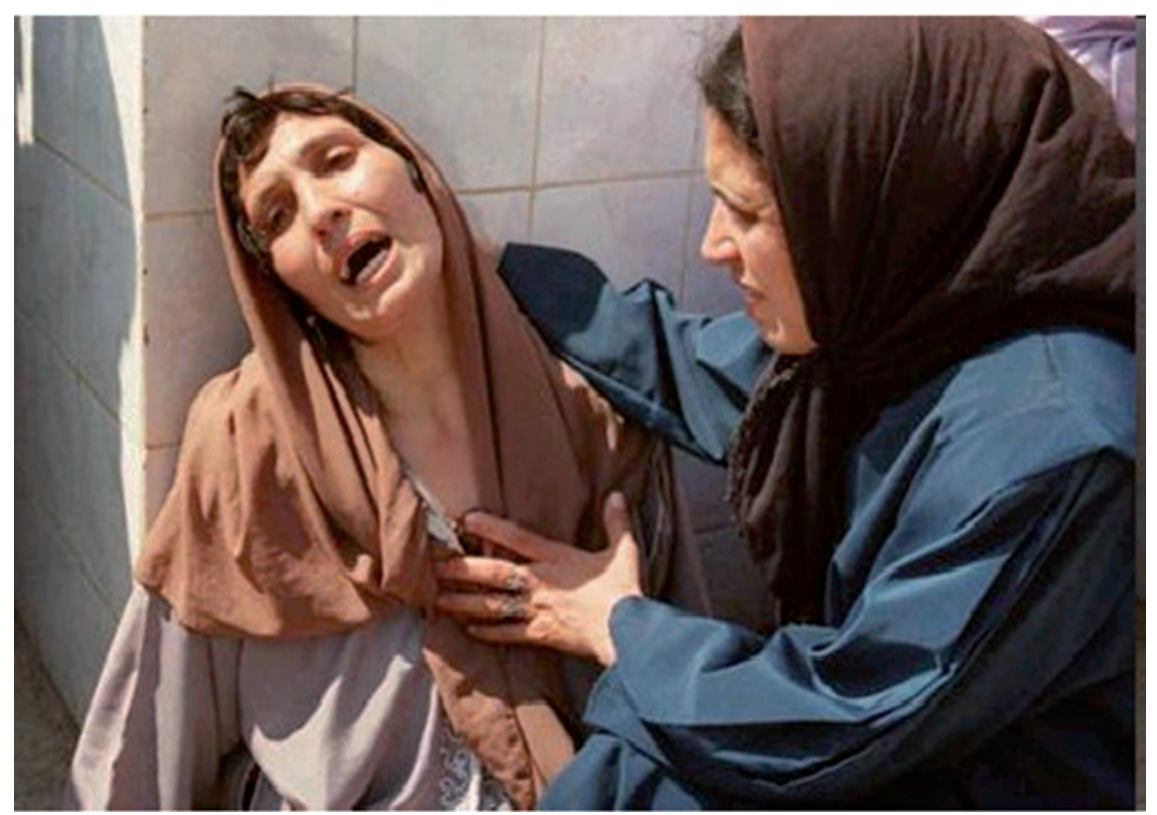

Figure 3 : Massacre à Bentalha, 1997, (C) AFP/ Hocine Zaoura

Comme Le Monde, Libération revient, dans le second temps médiatique et sémiotique, sur l'image de l'événement, par un commentaire de Gérard Lefort ${ }^{9}$

8. «Photogénie, fait remarquer le TLF, peut également désigner une qualité, une propriété de l'image: La photogénie est une propriété émouvante de l'image, à laquelle contribuent, non seulement le "don" de l'interprète, sa personnalité et celle du réalisateur qui le dirige, mais aussi des artifices techniques, grimage, éclairage, angle de prise de vue, plan, effets spéciaux », Giraud, 1956, p. 224.

9. Lefort, Gérard, 1997, «Une image unanime et ambigüe », encadré titré " Retour sur images », Libération, Médias, Jeudi 25 septembre. 
qui reprend l'argumentaire sur le photojournalisme en période de guerre, insérant un autre stéréotype discursif justifiant en partie les photographies de femmes dans ces circonstances : " c'est un fait de culture commun à toutes les civilisations méditerranéennes que la douleur des femmes y soit extrêmement visible ». Il interroge l'effet d'une telle photographie qui convoque, à notre mémoire et à notre conscience, " d'autres représentations de la peine au féminin » : le néo-réalisme du cinéma italien, avec les figures de la Magnani ou de la Callas dans le rôle de Médée, le style de Pasolini filmant l'Évangile selon saint Matthieu et, bien entendu, " en arrière-monde encore plus lointain ", la peinture et la sculpture du catholicisme avec la Mater dolorosa du Caravage et la Pietà de Michel-Ange : « la Vierge en somme ». Il souligne ainsi l'ambiguïté provoquée par l'irruption d'un imaginaire occidental dans le monde musulman.

La photographie fonctionne, selon cette perception interprétative, comme un décalque de l'icône de la Vierge, qui prend le statut d'image première, matricielle. C'est ainsi qu'un journaliste de Télérama ${ }^{10}$ évoque, à propos de la " dimension biblique de cette image ", " la pose de pietà de cette mère déchirée, le visage de marbre blanc sculpté par la douleur, comme une statue du Bernin », et « la couleur des drapés sortie d'un tableau de la Renaissance italienne ». On pourrait ajouter le corps affaissé sous le poids du malheur, dans une posture d'abandon à la souffrance, l'inclinaison de la tête, la mimique de la plainte et du cri, le geste d'affection de celle qui occupe la place de Marie-Madeleine, le jeu de la lumière zénithale qui modèle les vêtements et les traits des visages.

Si l'art chrétien prend son essor à partir de la période byzantine, de symboles en allégories, il faut attendre plusieurs siècles pour que se dégagent les thèmes dont s'inspirent les Madones occidentales : la Vierge en bénédiction, la Vierge et l'Enfant, la Vierge sur son trône. La codification symbolique des formes et des couleurs se met en place au Moyen-Âge, où domine le dogme théologique, et c'est au XIII ${ }^{\mathrm{e}}$ et $\mathrm{XIV}^{\mathrm{e}}$ siècle, alors que la Vierge est particulièrement vénérée, que le charnel investit la peinture et la sculpture religieuses, au détriment de la fonction d'image de dévotion. L'art chrétien évolue vers un style que l'on qualifie de pathétique, où prévalent les représentations de la Passion et la Crucifixion, avec la mise en scène des souffrances du Christ et la douleur de la Vierge, grande figure de la déploration.

À propos de l'exaltation, par les artistes du XV $\mathrm{XV}^{\mathrm{e}}$ siècle, de la figure de Marie, un historien d'art déclare : "Ils en ont fait un modèle et une consolation pour toutes les mères à qui un destin impitoyable - si l'on peut appeler destin cette faillite de l'humanité qu'est la guerre - réclamait, comme un sacrifice suprême, l'immolation de leur fils. " ${ }^{11}$ Un autre historien d'art, Germain Bazin, voyait, en 1941, dans la Pietà de Villeneuve-lès-Avignon d'Enguerrand Quarton (mais le tableau n'est pas encore attribué au peintre au moment où il écrit son texte), un autre exemple de sacrifice :

À l'heure angoissante où j'écris ces lignes, je voudrais voir en la Vierge de la Pietà d'Avignon l'image de la France meurtrie méditant sur ses morts et sa grandeur

10. Télérama, $\mathrm{n}^{\mathrm{O}} 2490,1^{\mathrm{er}}$ octobre 1997.

11. Hattinger, François, non daté, Madones italiennes, Payot Lausanne, p. 8. 
déchue. Loin de s'abandonner aux vains regrets, au lieu de s'avilir en « remâchant la nuit tous les aigres péchés du jour », je la vois, dans cette paralysie momentanée de ses forces, se recueillir, se concentrer, puiser dans le sacrifice l'énergie de vouloir son destin, et non de le subir, car elle se souvient de cette France du XV $\mathrm{X}^{\mathrm{e}}$ siècle, bafouée et sanglante, qui par la vertu du sacrifice d'un être pur, ressuscita, glorieuse de ses cendres. $^{12}$

L'époque où les images, qu'elles soient d'histoire ou de dévotion, étaient une invitation à la contemplation, en méditant les souffrances du modèle à des fins de conversion par transmutation des passions, semble bien définitivement révolue. L'appel à la compassion, présent dans la citation en latin tirée des Lamentations du prophète Jérémie (Lam. I, 12) et gravée sur le pourtour du fond d'or, encadrant la scène de la Pietà de Villeneuve-lès-Avignon : " Ô vous tous qui passez par ce chemin, regardez et voyez s'il est douleur comparable à la mienne ${ }^{13}$, ne semble pas plus propice, aujourd'hui, à un examen de conscience. Du moins celui-ci a changé de nature. La société médiatique contemporaine, qui exalte à sa façon les figures de la victime, est portée à l'exhibition télévisuelle des malheurs, par une nouvelle rhétorique des passions.

Et l'image en elle-même, dans sa conception, sa fabrication et son utilisation, est devenue un objet permanent de circulation, à grande vitesse, d'interprétation et de discussion. Elle est aussi l'objet d'une autre forme de consécration, documentaire, parfois publicitaire. On verra apparaître la photographie à la «Une »des numéros de fin d'année de nombreux hebdomadaires, français et étrangers, sous la légende des photographies de l'année, celles qui ont fait et récapitulent en images l'histoire récente, avant de rejoindre le panthéon des images du siècle ${ }^{14}$. Le montage médiatique de la mémoire imagée tend alors à déniveler l'interprétation, par alignement ou collage, provoquant d'autres significations collatérales. Nous versons alors dans le temps de la commémoration, orienté vers le futur, qui récapitule et dépasse les deux premiers temps, celui du présent d'actualité et celui des figures emblématiques d'une tradition culturelle. De nouveaux symboles sont créés, que l'on nomme "icônes ». Elle fera aussi l'objet, cet article en témoigne après d'autres, d'études, au titre d'exemplum, sur ce qu'Éliseo Véron appelait la semiosis sociale ${ }^{15}$.

\section{TRANSPOSITION}

À l'archive se surajoute un autre art de la mémoire, celui de l'artiste qui saisit les images d'actualité, investies par le regard pour leurs valeurs esthétiques, et les

12. Bazin, Germain, 1947, "La Pietà d'Avignon », in Les Trésors de la peinture française, Éditions d'Art Albert Skira, Genève.

13. En latin : O vos omnes qui transitis per viam attendite et videte si est dolor sicut dolor meus.

14. Par exemple dans Robin, Marie-Monique, 1999, Les 100 photos du siècle, Éditions du Chêne.

15. Voir Véron, Éliseo, 1987, La semiosis sociale. Fragments d'une théorie de la discursivité, PUV, SaintDenis. La photographie de Zaouar a fait l'objet d'un article : Delannoy, Pierre-Alban, 1998, « L'image et le savoir partagé : la Pietà de Bentalha », in Communication \& langages, 115, pp. 52-57, puis d'un bref ouvrage du même auteur : Delannoy, Pierre-Alban, 2005, La Pietà de Bentalha. Étude du processus interprétatif d'une photo de presse, L'Harmattan, Paris. 
déplace dans le champ de l'art en créant une œuvre plastique, qui est une forme de monument donnée à voir et à percevoir dans l'espace muséal. C'est le geste opéré par Pascal Convert, dont le travail vise à « interroger cette vaste empathie au monde à travers les images ${ }^{16}$. Il explore en permanence le flux médiatique et capte ce qu'il appelle des "stéréotypes forts ", des "photos qui portent une généalogie lourde, évoquent l'histoire de l'art tout en parlant de l'actualité, ouvrent des débats riches ${ }^{17}$. La sémiose fait alors un tour supplémentaire par une réinterprétation de l'image-icône, avec sa double référence. Cette réinterprétation est d'abord formelle, par la technique du modelage et du moulage - l'empreinte - et l'utilisation d'un matériau, la cire, avec ses qualités organiques, ses connotations religieuses et son usage dans les rites mortuaires, qui se conjuguent pour restituer à la représentation la double dimension de réalité sensible et de symbole.

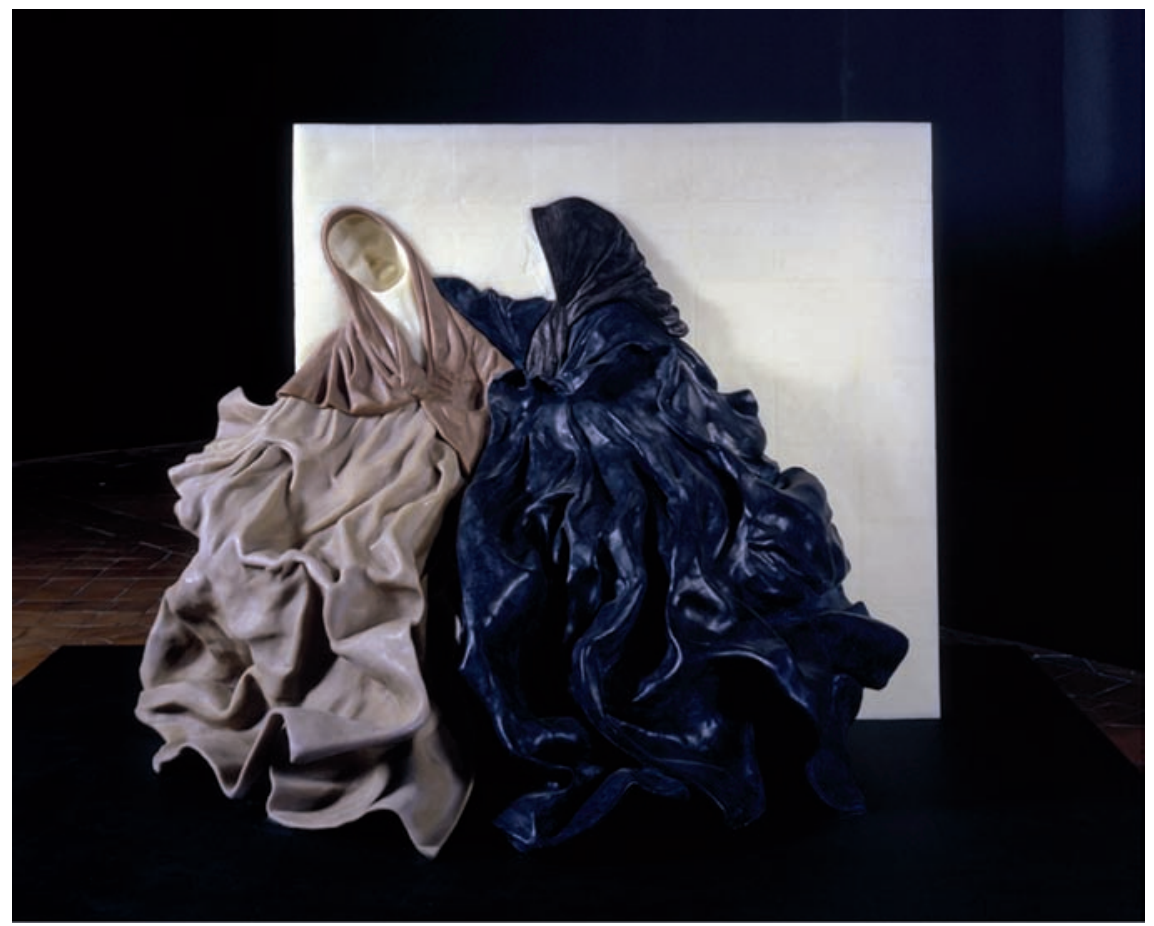

Figure 4 : Pascal Convert, Madone de Bentalha (d'après la photographie Massacre à Bentalha d'Hocine Zaourar/AFP, Prix World Press Photo de l'année 1997), 2001-2002, Cire polychrome, $220 \mathrm{~cm} \times$ $250 \mathrm{~cm} \times 40 \mathrm{~cm}$. Photo : Frédéric Delpech, (C) Pascal Convert/SODRAC (2009), Collection musée d'Art moderne Grand-Duc Jean, Mudam Luxembourg

Au festival d'arts visuels Printemps de septembre de Toulouse, en 2004, Pascal Convert a présenté trois sculptures monumentales en cire, installées au réfectoire des Jacobins. Elles ont été réalisées, avec l'assistance de deux sculpteurs du musée

16. Guerrin, Michel, 2004, « Pascal Convert redonne forme aux images », Le Monde, jeudi 30 septembre, p. 27.

17. Ibid. 
Grévin, à partir de trois images de presse : une photographie de Georges Mérillon (1990) : femmes pleurant un mort au Kosovo ; la photographie d'Hocine Zaouar (1997) ; un photogramme tiré d'une scène filmée dans la bande de Gaza par Talal Abou Rahmeh (2000) : la mort du petit Mohammed, un enfant de 12 ans. Les œuvres sont l'acquisition du musée d'Art moderne du Luxembourg. La Madone de Benthala a fait l'objet d'une exposition à la galerie de l'UQUAM, dans le cadre du mois de la photographie à Montréal, en septembre-octobre 2009. Il faut préciser que Pascal Convert associe, dans les expositions, les sculptures avec les photographies, qui sont comme des originaux dont elles seraient des répliques, par transposition, et un montage de documents visuels et filmiques qui témoignent de l'événement référentiel et du contexte de la création.

Dans un entretien de 2003, lors de l'exposition annuelle Visa pour l'image :

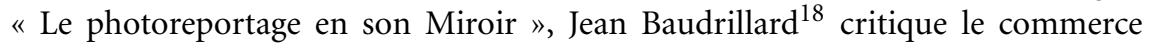
économique et politique des images médiatiques, avec l'exploitation publique qui est faite du malheur et de la douleur, la surcharge de signes qui est imposée aux photographies, évoquant notamment « la madone algérienne, dont le fétichisme est proche des images de stars ». À la question de savoir comment délester les images de presse du trop-plein de significations pour en retrouver l'affect propre, il répond en disant qu'il faut " un transfert poétique », et qu'il faut du vide entre les photos et dans les photos. S'il est vrai que l'écart est constitutif de l'activité symbolique et que la fonction de l'art est de décaler notre perception du monde, le redoublement apparent effectué par les œuvres de Pascal Convert a pour effet d'annuler la croyance en l'image comme doublure. En ce sens, l'œuvre d'art, par défiguration, au-delà de la ressemblance, est un retour à ce réel impossible à représenter.

Mais le regard peut-il s'abstraire de la perception culturelle et de la reconnaissance du déjà-vu et du déjà-dit ? Dans un dossier publié par la revue art press en $2003^{19}$ et intitulé «Images d'Algérie », Pascal Convert raconte son voyage en Algérie en 2002 et sa rencontre avec Hocine. Dans un retour sur les analogies établies entre la photographie et certains tableaux de la tradition chrétienne, il traverse cet écran et voit dans le visage de la Madone et son cri de douleur muet un possible appel à la « vengeance meurtrière ». C'est alors l'image tragique de Médée, la meurtrière par vengeance, qui surgit : «Une fascinante et obtuse bouche nous fait passer de l'iconographie chrétienne et son imagerie de la douleur à la tragédie grecque et sa rhétorique de la vengeance. La Mater Dolorosa devient Médée. ${ }^{20}$ L'article est intitulé «La Médée algérienne ». Le modèle grec, avec son cycle de vengeances et de trahisons, ouvre ainsi son regard à une autre compréhension de l'histoire contemporaine de l'Algérie. Le renvoi qu'il fait à la notion de «sens obtus » chez Barthes ${ }^{21}$ marque l'altération du sens par certains éléments qui « dérangent », les signifiants qui sont en trop ; le sens obtus « peut être vu comme

18. Baudrillard, Jean, 2003, «Le photoreportage en son Miroir », Le Monde, samedi 30 août, p. 24.

19. Convert, Pascal, 2003, «Images d'Algérie », art press, $\mathrm{n}^{\circ} 386$.

20. Ibid., p. 20.

21. Barthes, Roland, 1994, "Le troisième sens. Notes de recherche sur quelques photogrammes de S. M. Eisenstein », [Cahiers du Cinéma, juillet 1970] Euvres complètes Tome II, Paris, Seuil. 
un accent, la forme même d'une émergence, d'un pli (voire d'un faux pli) dont est marquée la lourde nappe des informations et des significations. ${ }^{22}$ On pourrait parler de signifiants diacritiques, non superflus, puisqu'ils servent à sélectionner une autre valeur, qui (dés)oriente la lecture et l'interprétation. Mais alors que Barthes voit dans le "sens obtus ", «à la fois têtu et fuyant », une échappée hors de ce qui constitue le "sens obvie » : le savoir partagé, l'intention de signification, la codification, le déplacement qui est ici opéré par l'observateur-interprète n'annule pas le premier geste de l'artiste et le sens déjà-là, il est le signe du surinvestissement culturel du regard sur une image en vision stéréoscopique.

\section{DOMINIQUE DUCARD}

